\title{
Normal nasal mucociliary clearance in CF children: evidence against a CFTR-related defect
}

\author{
D. McShane*,\#, J.C. Davies*,\#,+, T. Wodehouse*,+, A. Bush ${ }^{\#}$, D. Geddes ${ }^{\uparrow}$, E.W.F.W. Alton*,ף,+
}

Normal nasal mucociliary clearance in CF children: evidence against a CFTR-related defect. D. McShane, J.C. Davies, T. Wodehouse, A. Bush, D. Geddes, E.W.F.W. Alton. (C) ERS Journals Ltd 2004.

ABSTRACT: Studies on mucociliary clearance (MCC) in cystic fibrosis (CF) have produced conflicting results. This study aimed to differentiate primary (ion transportrelated) from secondary (inflammatory) causes of delayed MCC in CF.

Nasal MCC was measured in 50 children (CF, primary ciliary dyskinesia (PCD) and no respiratory disease). Nasal lavage fluid was analysed for interleukin (IL)-8 and tumour necrosis factor- $\alpha$. Similar measurements were obtained in adult $\mathrm{CF}$ patients with and without chronic sinusitis (CS).

Children with CF had neither delayed MCC nor increased levels of cytokines. Conversely, children with PCD had prolonged MCC times (all $>30 \mathrm{~min}$ ) and significantly raised levels of IL-8. CS-positive CF adults had significantly slower MCC than CS-negative subjects, but IL-8 levels were low and similar in both groups.

Decreased airway surface liquid and delayed mucociliary clearance are the postulated primary mechanisms in cystic fibrosis. However, the current study reports that cystic fibrosis children have normal nasal mucociliary clearance. Abnormalities appeared in cystic fibrosis adults with symptoms of chronic sinus disease, suggesting a secondary rather than primary phenomenon. Studies to explore this mechanism in the distal, more sparsely-ciliated airways could aid an understanding of pathogenesis and the development of new treatments.

Eur Respir J 2004; 24: 95-100.
*Dept of Gene Therapy, Imperial College at the National Heart and Lung Institute, Depts of \#Paediatric Respiratory Medicine and Thoracic Medicine Royal Brompton Hospital, and ${ }^{+}$UK Cystic Fibrosis Gene Therapy Consortium, London, UK.

Correspondence: J. Davies, Dept of Gene Therapy, National Heart and Lung Institute, Manresa Road, London SW3 6LR, UK.

Fax: 442073518340

E-mail: j.c.davies@ic.ac.uk

Keywords: Airway inflammation cystic fibrosis

mucociliary clearance

nasal lavage

paediatric

Received: August 272003

Accepted after revision: February 202004
The mucociliary escalator is one of the primary innate defence mechanisms of the lung, playing a central role in pulmonary health. It consists of two intrinsically linked components: the ciliated epithelium and the airway surface liquid (ASL); the combined function of which is efficient removal of foreign material and cellular debris from the lungs. The ciliated epithelium extends from the inferior turbinate in the nose to the distal small airways. It is covered by the ASL, which is composed of two distinct layers: the periciliary liquid (PCL) and the mucus layer [1]. The bodies of the cilia are surrounded by the PCL, which is approximately the same height as an outstretched cilium, whilst the tips are in contact with the overlying layer of mucus. The mucus layer consists of heavily glycosylated macromolecules known as mucins that behave as a tangled network of polymers, binding and trapping inhaled particles to facilitate clearance from the lung [2]. In contrast, the PCL is of low viscosity and provides the medium in which the cilia beat, propelling the overlying mucus layer in a caudal to cranial direction. Normal composition and depth of the PCL is thought to be crucial in this mechanism. Thus, optimal mucociliary clearance (MCC) involves the interaction of the airway epithelium including the cilia, the PCL (which must be of normal depth and chemical composition), and airway mucus of normal composition and rheology.

Cystic fibrosis (CF) is a multi-system disorder characterised by chronic bacterial airway infection, excessive airway inflammation and eventual bronchiectasis. The pathophysiology of $\mathrm{CF}$ lung disease remains uncertain, although prolonged MCC appears to be central to most current hypotheses [3]. The primary defect in CF is either absence or reduced function of the cystic fibrosis transmembrane conductance regulator (CFTR) protein, a cAMP-dependent chloride channel, which results in abnormal ion transport across the apical surface of epithelial cells [4]. CFTR is expressed in many epithelial tissues [5], including those of the upper and lower airways, pancreas, sweat duct and gastrointestinal system. However, the lack of normal function within the lower airway causes the greatest degree of morbidity and mortality [6].

In addition to its function as a chloride channel, CFTR has other functions, including an inhibitory effect on the epithelial sodium ion channel [7]. The low-volume hypothesis of disease pathogenesis suggests that a lack of normal CFTR function within the $\mathrm{CF}$ airway leads to increased sodium and water absorption resulting in reduced ASL volume $[3,8,9]$. Reduction in volume of the PCL results in the cilia becoming embedded in the dehydrated mucus, uncoupling of the ciliamucus interaction and impaired MCC [3]. This gluing or "velcro" effect between mucins also inhibits cough clearance. TARRAN et al. [9] have confirmed in vitro that ASL volume is regulated by active ion transport. Additionally, the authors showed that the mucus layer acts as a reservoir with bidirectional flow of fluid between it and the PCL to maintain optimal volume for ciliary activity and active mucus transport. In $\mathrm{CF}$, however, disruption of ASL volume regulation could mean that the fluid debt of the PCL exceeds the capacity of the mucus layer to buffer it. Thus, one can 
postulate that $\mathrm{CF}$ lung disease results from depletion of the ASL volume and impaired MCC. Inhaled pathogens fail to be cleared and chronic infection ensues. Airway damage arises from a combination of toxic bacterial products [10, 11] and an exaggerated host inflammatory response [12]. In addition to the hydration of the ASL, there may be other factors which could impair $\mathrm{MCC}$ in $\mathrm{CF}$. For example, $\mathrm{CF}$ mucins are known to be over-sulphated [13]. Following infection, CF sputum becomes increasingly viscous due to its high extracellular DNA content [14], whilst various exoproducts of the common bacterial pathogen, Pseudomonas aeruginosa, may slow ciliary beat frequency and disrupt ciliary orientation [10].

Despite this hypothesis and the supporting in vitro data, clinical studies of MCC in the upper and lower airways of $\mathrm{CF}$ subjects have produced conflicting results. Some studies report normal $\mathrm{MCC}$ in $\mathrm{CF}[15,16]$, others demonstrate similar abnormalities in $\mathrm{CF}$ and other disease groups [17, 18] or impaired MCC in CF patients [19-24], leading to uncertainty as to whether any observed impairment is related to the low volume hypothesis or results from "secondary" phenomena such as inflammation. The adverse effect of inflammatory damage has been confirmed in studies of nonCF bronchiectasis [17, 18], asthma [24] and chronic sinonasal disease $[17,25]$. The majority of $\mathrm{CF}$ studies have been performed in adults; sinusitis is common in this age group [26], and distinction of a primary from secondary effect is therefore difficult. Such differentiation could have important therapeutic implications, for example, in the timing of interventions designed to improve $\mathrm{MCC}[27,28]$ or reduce inflammation [29]. The present authors attempted to clarify this issue by measuring $\mathrm{MCC}$ in children with and without $\mathrm{CF}$, and in $\mathrm{CF}$ adults with and without chronic sinusitis (CS).

\section{Methods}

\section{Subjects}

Paediatric subjects were recruited from the outpatient department of the Royal Brompton Hospital. Three groups of children were studied, as follows. 1) CF: 18 children (eight males, median (range) age 11 yrs (9.5-15)) who had been diagnosed on conventional biochemical or genetic testing [30] (10 homozygous and seven heterozygous for the $\Delta \mathrm{F} 508$ mutation; in the remaining $\mathrm{CF}$ child, neither mutation was identified). 2) Primary ciliary dyskinesia (PCD): 11 children (seven males, age 12 yrs (9-14.7)) whose diagnosis had been established on nasal brush biopsy [31] were included as positive controls for delayed MCC. 3) No respiratory disease (NRD): 21 children (13 males, age 12 yrs (9.5-16)) with simple non-cyanotic cardiac disease without dextrocardia were recruited as negative controls. Previous work has shown no increase in the incidence of ciliary abnormalities in this group [32].

Twenty-eight adults with CF (17 males; age 23 yrs (16-55)) were recruited from the outpatient department of the Royal Brompton Hospital, using similar exclusion criteria to those above. None of the subjects studied admitted to smoking. Nine CF subjects were homozygous and seven heterozygous for the $\Delta \mathrm{F} 508$ mutation. CFTR genotype was unknown in 12 subjects. Subjects were determined to have chronic sinusitis (CS +ve) or not (CS -ve) by a validated symptom questionnaire (see Appendix 1) [33]. Exclusion criteria for both groups included the presence of nasal polyps (determined by clinical examination by an experienced clinician on the day of testing) or intercurrent upper respiratory tract infection. The study was approved by the Ethics Committee of the Royal Brompton and Harefield NHS Trust and subjects or parents gave informed consent.

\section{Measurement of nasal mucociliary clearance}

MCC was measured by the saccharin clearance technique $[34,35]$. After subjects had blown their nose clear of secretions, a 2-mm diameter piece of saccharin was placed with Hartmann's crocodile forceps at the anterior edge of the inferior turbinate under direct vision through an auroscope. Subjects were then asked to sit forward, breathe through their mouth, not to sniff and to swallow normally. The time taken from placement of the saccharin to tasting on the back of the tongue was recorded. The test was abandoned for reasons of practicality at $30 \mathrm{~min}$ in children; in adult subjects the test was abandoned after $60 \mathrm{~min}$. In these subjects, ability to taste saccharin was confirmed subsequently by placing a piece directly onto their tongue.

\section{Collection of nasal lavage specimens}

Nasal lavage fluid (NLF) was collected from the opposite nostril upon completion of the saccharin test using a technique described by other investigators [36]. Leaning forward, subjects had a soft, size- 6 French gauge nasogastric tube (Meddis Ltd, Wallingford, UK) inserted into the nostril to a depth of $4 \mathrm{~cm}$. The nasal cavity was then washed slowly with $4 \mathrm{~mL}$ of sterile phosphate buffered saline (Sigma, Poole, UK) and the effluent was collected in a sterile universal container held at the edge of the nostril.

\section{Processing of the nasal lavage specimens}

All nasal lavage specimens were immediately placed on ice. A sample of fluid was sent for routine microbiological culture (Clinical Microbiology Laboratory, Royal Brompton Hospital, London, UK). As soon as possible, specimens were centrifuged at $2,000 \times g$ for $15 \mathrm{~min}$. The supernatant was divided into $1-\mathrm{mL}$ aliquots and stored at $-80^{\circ} \mathrm{C}$ for future analysis. Samples were subsequently analysed for interleukin (IL)- 8 and tumour necrosis factor (TNF)- $\alpha$ by ELISA according to the manufacturer's instructions (R\&D Systems, Abingdon, UK).

\section{Statistics}

Data were nonparametrically distributed and are reported as median (interquartile range). For clarity, age data are presented as median (range). The Kruskal-Wallis test and the Mann-Whitney U-test (with corrections for multiple comparisons where appropriate) were used to compare groups and the null hypothesis was rejected at $\mathrm{p}<0.05$. For the purposes of statistical analysis, subjects having failed to taste the saccharin by the cut-off times of 30 (children) or 60 (adults) min had times recorded as 30 or $60 \mathrm{~min}$.

\section{Results}

\section{Paediatric study}

No difference was seen in MCC times between the children with CF (11 min (8-15)) and those with no respiratory disease (11 min (9-16)), whereas those with PCD all had delayed 


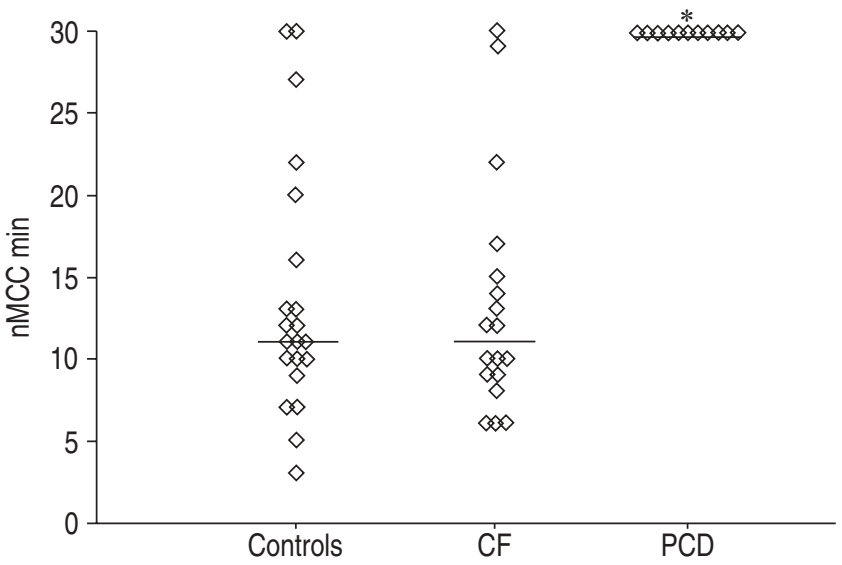

Fig. 1. - Nasal mucociliary clearance (nMCC) was measured in three groups of children (controls $(n=21)$, cystic fibrosis $(\mathrm{CF})(\mathrm{n}=18)$ and primary ciliary dyskinesia (PCD) $(\mathrm{n}=11))$ using the saccharin technique. No difference was found between the controls and CF groups. All children with PCD had prolonged nMCC ( $>30$ min, marked on the graph at $30 \mathrm{~min}$ for convenience). Bars represent median values. *: $\mathrm{p}<0.05$ when compared to both other groups.

MCC ( $>30$ min; $p<0.05$ compared with both other groups; fig. 1). Within the $\mathrm{CF}$ group, there was no difference between subjects homozygous for the $\Delta \mathrm{F} 508$ mutation and the rest of the group $(\Delta / \Delta 11 \mathrm{~min}(9-24)$; other $11 \mathrm{~min}(6-13) ; \mathrm{p}=0.6)$.

There was no evidence of excess inflammation in the $\mathrm{CF}$ NLF as assessed by levels of the neutrophil chemoattractant, IL-8 (CF $20.5 \mathrm{pg} \cdot \mathrm{mL}^{-1} \mathrm{NLF}(0-43.6)$; NRD $0 \mathrm{pg} \cdot \mathrm{mL}^{-1} \mathrm{NLF}$ (0-76.4); $p=1.0$; fig. 2). Interestingly, however, the group with PCD had significantly raised levels of IL-8 $\left(304.8 \mathrm{pg} \cdot \mathrm{mL}^{-1}\right.$ NLF (102.8-512.3); $\quad \mathrm{p}<0.01$ compared with both other groups). NLF TNF- $\alpha$ was below the limit of detection in all samples. There was only one positive microbiological culture: Streptococcus pneumoniae from a child with PCD. The IL-8 level in this subject was not, however, amongst the highest in the PCD group at $244 \mathrm{pg} \cdot \mathrm{mL}^{-1}$.

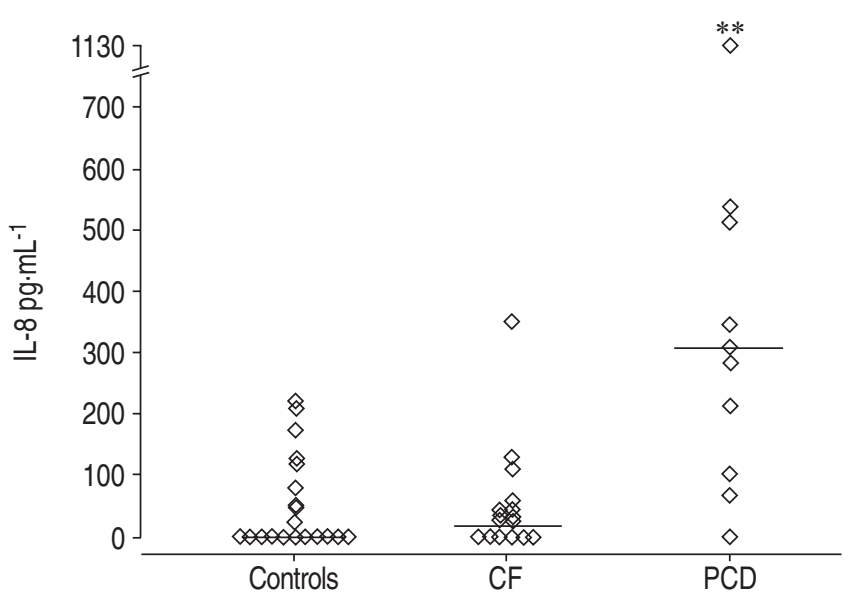

Fig. 2.-The interleukin (IL)-8 content of nasal lavage fluid (NLF) was measured by ELISA according to the manufacturer's instructions. There was no evidence of increased inflammation in either the control $(n=21)$ or cystic fibrosis $(C F)(n=17)$ groups. However, the IL-8 content of NLF from primary ciliary dyskinesia (PCD) $(n=11)$ children was significantly raised compared with both other groups. Bars represent median values. **: $\mathrm{p}<0.01$.

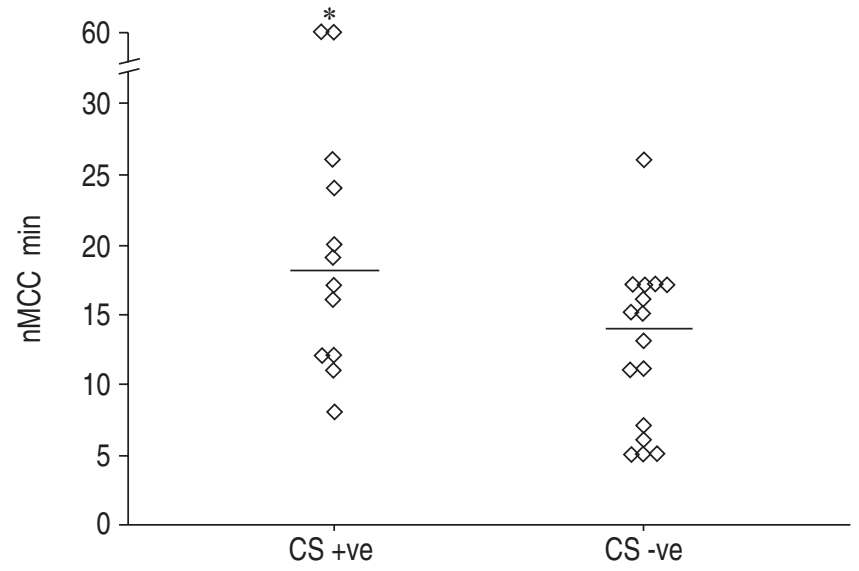

Fig. 3. - Nasal mucociliary clearance (nMCC) in cystic fibrosis $(\mathrm{CF})$ adults with $(\mathrm{CS}+\mathrm{ve})(\mathrm{n}=12)$ and without $(\mathrm{CS}-\mathrm{ve})(\mathrm{n}=16)$ a history of chronic sinus disease. Patients who had not tasted the saccharin by the 60-min cut-off point are marked on the graph at $60 \mathrm{~min}$ for convenience. Bars represent median values. *: $\mathrm{p}<0.05$.

\section{Adult study}

Twelve of the CF subjects (43\%) fulfilled the criteria for chronic sinusitis $(\mathrm{CS}+\mathrm{ve})$. They had significantly $(\mathrm{p}<0.02)$ longer MCC times (18 min (11.5-24)) than the $16 \mathrm{CS}-\mathrm{ve}$ subjects (14 min (6-16.8); fig. 3). However, NLF IL-8 was below the limit of detection in the majority of subjects, with no significant differences between $\mathrm{CS}+\mathrm{ve}\left(0 \mathrm{pg} \cdot \mathrm{mL}^{-1}\right.$ $(0-38.8))$ and CS-ve $\left(0 \mathrm{pg} \cdot \mathrm{mL}^{-1}(0-0)\right)$ adults (fig. 4). As in the paediatric study, TNF- $\alpha$ was undetectable in all samples. There were no positive microbiology cultures.

\section{Discussion}

Nasal MCC was assessed in an attempt to distinguish a primary, low ASL volume-driven impairment in MCC in $\mathrm{CF}$ subjects from a secondary effect of mucosal inflammation, such as that reported in subjects with sinonasal disease. Normal MCC in childhood, early in the course of $\mathrm{CF}$, and impairment later, in adults with a history of $\mathrm{CS}$, was

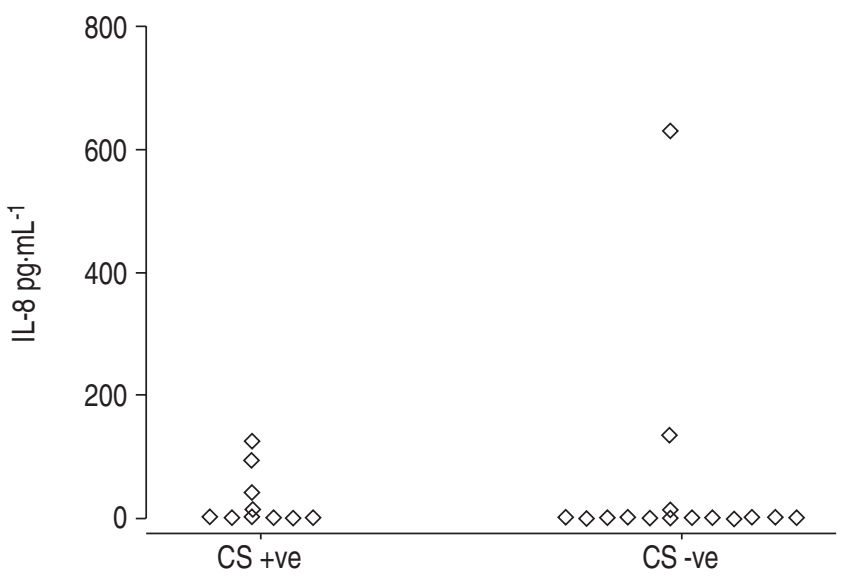

Fig. 4. - No evidence of excess inflammation interleukin (IL)-8 was found in the nasal lavage fluid (NLF) of cystic fibrosis (CF) subjects with chronic sinusitis $(\mathrm{CS}+\mathrm{ve})(\mathrm{n}=12)$ as compared to those without $(\mathrm{CS}-\mathrm{ve})(\mathrm{n}=16)$ 
demonstrated. Using the technique of nasal lavage, it could not be confirmed directly that slowing of MCC was related to an inflammatory process; levels of cytokines in NLF being mostly below the limits of detection. This may be due either to a technical problem, such as the variable dilution factor inherent in lavage sampling, or to the fact that the inflammation related to chronic sinus disease does not cause inflammatory proteins to be secreted onto the airway surface. However, these results suggest, at least for the large proximal extensively ciliated airways, that reduced ASL volume related to sodium and water hyperabsorption does not directly impact on MCC, and they provide some preliminary evidence to suggest that this may be due to inflammation.

The data in support of low ASL as the triggering event for this cascade come mainly from in vitro and animal work. MATSUI et al. [3] reported that in highly differentiated cultured CF monolayers, ASL composition was normal, but rates of fluid absorption were increased. After 24-h culture, ASL volume was significantly depleted and rotational mucus transport had ceased on CF cells. TARRAN et al. [8] obtained murine nasal tissue after in vivo osmium tetroxide fixation and demonstrated a significant reduction in ASL height from $7 \mu \mathrm{m}$ (the height of an extended cilium) in wild-type mice to less than $5 \mu \mathrm{m}$ in CFTR ${ }^{\text {tm1Unc }} \mathrm{CF}$ mice. Interestingly, the authors also reported goblet cell meta- and hyperplasia, which they hypothesise was secondary to intermittent infection and inflammation caused by impaired clearance, although MCC was not measured. In a recent report, however, this group has reported normal MCC in both the upper and lower airways of CF mice [37]. ZAHM et al. [38] reported slower tracheal MCC in CFTR ${ }^{\text {tm1 } 1 \text { Unc }} \mathrm{CF}$ mice, but also showed significant inflammation in the lamina propria. These mice had not been exposed to infection, supporting the possibility of primary inflammation. In contrast, MCCRAY et al. [39] studied cultured tracheal epithelium from $\Delta \mathrm{F} 508$ homozygous mice with a radiotracer technique and reported normal ASL height and composition. These mice did not have evidence of inflammation on bronchoalveolar lavage (BAL). Thus, these somewhat conflicting data, whilst largely favouring low volume ASL in CF, do not confirm that this abnormality in isolation sufficiently impairs MCC in humans to produce lung disease.

Human studies do not completely clarify the situation either. Direct measurements of ASL have proved extremely difficult clinically and, to date, no studies have measured ASL depth in situ in the human airway. Many groups have measured MCC in CF, both in the nose and the lung [40]; the majority report that $\mathrm{CF} \mathrm{MCC}$ is slow, but others found no impairment and, importantly, similar reductions in MCC rates have been reported in other pulmonary disease states, including non- $\mathrm{CF}$ bronchiectasis and asthma, suggesting a role for nonspecific mechanisms. None of the studies have focused on the early stages of $\mathrm{CF}$ disease, when the influences of infection and inflammation might be reduced, and thus the question of a primary versus a secondary impairment in MCC remains unanswered.

The choice of the nose for this study was based on several factors, including the relatively noninvasive nature of the test, the ability to study young children with ease, and, most importantly, the relative lack of pathology at this site, at least early in the course of the disease. Clearly, a limitation of this study is extrapolation of these findings to the distal airways, where $\mathrm{CF}$ disease is thought to begin. There are several possible reasons why either ASL volume, MCC or both, may differ at the two sites. First, both the epithelial cell composition and origin of ASL differ. Ciliated cells are less abundant in the distal airways, where they are interspersed with nonciliated epithelium, contrasting with the almost completely ciliated surface of the nose. ASL probably originates from the submucosal glands and sinuses in the nose, whereas in the distal airways, most probably arises from the bronchiolar epithelium itself or that of the alveolar region. Thus, it is possible that in the nose, the relatively large gland/ sinus output and the short distance for surface mucus clearance offsets the defect in the CF superficial epithelium for volume homeostasis. In contrast, in the bronchiolar region, the absence of glands, larger surface area and incompletely ciliated surfaces may demand more volume regulation by the superficial epithelium, and hence a greater expression of the defect in CF airways. A second possible explanation for the present findings is the release of nucleotides, including ATP, from airway epithelium in response to a variety of stimuli, such as airflow-induced sheer stretch [41]. These nucleotides initiate chloride and volume secretion, which was demonstrated in vitro to significantly increase ASL height. Thus, in contrast to the results generated in static air/liquid interface studies, ASL volume in vivo may be maintained in part by other mechanisms.

Lung studies of MCC in early childhood would contribute significantly to this area of research. However, the practicalities involved in such studies, including the requirement for cooperation in the inspiratory technique, the radiation and study times involved, and the ethical consideration of recruiting controls, have limited the majority of studies to older children and adults. Even in this cooperative group, the significant problems which have been reported, including coughing, lack of standardisation of particle deposition and differing methods of particle administration and image analysis, are likely contributors to the varying results in subjects with $\mathrm{CF}$. Although advances are being made, including the development of three-dimensional scanning facilities, these are not, as yet, widely available. At present, it is unlikely that reliable data can be generated in children $<7$ yrs. It is well recognised that by this age, even apparently mildly affected CF patients have significant lower airway inflammation and infection [42, 43], which would, therefore, confound a study of primary versus secondary MCC impairment.

These concerns led the current authors to examine MCC in the nose and to correlate these measurements with inflammation. Previous studies have confirmed the repeatability of the saccharin clearance technique in children and adults $[20,36$, 44], and this test is in clinical use in the present authors' institution. Sinonasal disease is uncommon in young CF children and, in line with others [45], the current authors did not show increased levels of the pro-inflammatory cytokine, IL-8, in nasal lavage samples. However, the majority of adult subjects, including those with histories suggestive of chronic sinus disease, also had low levels of this cytokine, highlighting possible limitations of the technique. Such limitations are well recognised, in particular the variable and uncertain yield of ASL volume. Some studies have utilised a dilution factor such as inulin for these purposes [46], although this approach is also problematic and is no longer recommended in guidelines for BAL [47], where similar concerns exist. Computed tomography scans were not used to assess sinus disease, due to the almost widespread opacities observed in older $\mathrm{CF}$ patients, likely related to obstruction [27], and the ethical issues of imaging purely for research purposes. Similarly, nasal biopsy, which may have significantly aided identification of epithelial or subepithelial inflammation, was deemed excessively invasive for this study, particularly in the paediatric population. Thus, although the relationship with disease stage and history of sinusitis support the current authors' hypothesis that impaired nasal MCC occurs secondary to inflammatory changes, further studies with increased numbers, strictly defined criteria for the diagnosis 
of sinus disease and more sensitive techniques to detect inflammation may be warranted. Such studies would also enable the possibility of extremely subtle derangements of MCC to be excluded in the paediatric CF cohort, for which this study may have been underpowered.

It was surprising to detect such high levels of IL-8 in the NLF of children with PCD, despite the fact that the majority had negative bacterial cultures. These children were initially recruited as positive controls for prolonged MCC and, although chronic rhinitis is a major feature of the disease [48], nasal lavage studies have not, to the current authors' knowledge, been previously reported. Despite the global defect in MCC, these patients are generally less severely affected than those with $\mathrm{CF}$, possibly due to preservation of cough clearance, although very few data exist on the extent of airway inflammation. There has been a link with low levels of nitric oxide [49], a molecule with known antibacterial properties, although this is also a feature of CF. Thus, this finding is currently unexplained and will be explored further in future studies.

In conclusion, the current study suggests that in the proximal, highly ciliated airways, defective cystic fibrosis transmembrane conductance regulator function alone does not impair mucociliary clearance. However, these findings may not be replicated in the distal, poorly ciliated small airways where cystic fibrosis pathology is thought to begin. Novel methodology to quantify mucociliary clearance and cough clearance in this site in young children or newborn infants before the onset of inflammatory changes, which may confound the picture, is urgently required. Understanding the sequence of events leading to early lung damage in cystic fibrosis infants could significantly influence the direction of development of new therapeutic approaches for this disease.

\section{Appendix 1: Rhinosinusitis assessment}

All $\mathrm{CF}$ adult subjects completed this questionnaire prior to undertaking the study. The investigator was blinded to its results. The diagnosis of chronic sinusitis (CS +ve) was made if subjects reported two or more symptoms from questions 4-8 on most days for $>6$ months.

1. Do you suffer from hayfever?

2. Are you allergic to cats, dogs or house dust mites?

3. Do you take any nasal medications?

4. Do you suffer with a post-nasal drip (catarrh) at the back of your throat?

5. Do you suffer from nasal congestion/blockage?

6. Do you suffer from frontal facial pain/headache?

7. Do you have difficulty lifting objects from the ground due to facial pressure?

8. Do you lose the sensation of taste?

\section{References}

1. Knowles MR, Boucher RC. Mucus clearance as a primary innate defense mechanism for mammalian airways. J Clin Invest 2002; 109: 571-577.

2. Kim KC, McCracken K, Lee BC, et al. Airway goblet cell mucin: its structure and regulation of secretion. Eur Respir $J$ 1997; 10: 2644-2649.

3. Matsui H, Grubb BR, Tarran R, et al. Evidence for periciliary liquid layer depletion, not abnormal ion composition, in the pathogenesis of cystic fibrosis airways disease. Cell 1998; 95: 1005-1015.

4. Welsh MJ. Abnormal regulation of ion channels in cystic fibrosis epithelia. FASEB J 1990; 4: 2718-2725.
5. Trezise AE, Buchwald M. In vivo cell-specific expression of the cystic fibrosis transmembrane conductance regulator. Nature 1991; 353: 434-437.

6. Koch C, Hoiby N. Pathogenesis of cystic fibrosis. Lancet 1993; 341: 1065-1069.

7. Ismailov II, Awayda MS, Jovov B, et al. Regulation of epithelial sodium channels by the cystic fibrosis transmembrane conductance regulator. J Biol Chem 1996; 271: 47254732.

8. Tarran R, Grubb BR, Parsons D, et al. The salt controversy: in vivo observations and therapeutic approaches. Mol Cell 2001; 7: 888-889.

9. Tarran R, Grubb BR, Gatzy JT, Davis CW, Boucher RC. The relative roles of passive surface forces and active ion transport in the modulation of airway surface liquid volume and composition. J Gen Physiol 2001; 118: 223-236.

10. Munro NC, Barker A, Rutman A, et al. Effect of pyocyanin and 1-hydroxyphenazine on in vivo tracheal mucus velocity. J Appl Physiol 1989; 67: 316-323.

11. Kanthakumar K, Taylor GW, Cundell DR, et al. The effect of bacterial toxins on levels of intracellular adenosine nucleotides and human ciliary beat frequency. Pulm Pharmacol 1996; 9: 223-230.

12. Muhlebach MS, Stewart PW, Leigh MW, Noah TL. Quantitation of inflammatory responses to bacteria in young cystic fibrosis and control patients. Am J Respir Crit Care Med 1999; 160: 186-191.

13. Cheng PW, Boat TF, Cranfill K, Yankaskas JR, Boucher $\mathrm{RC}$. Increased sulfation of glycoconjugates by cultured nasal epithelial cells from patients with cystic fibrosis. J Clin Invest 1989; 84: 68-72.

14. Shak S, Capon DJ, Hellmiss R, Masters SA, Baker CL. Recombinant human Dnase I reduces the viscosity of cystic fibrosis sputum. Proc Natl Acad Sci USA 1990; 87: 91889192.

15. Rayner CFJ, Rutman A, Dewar A, Cole PJ, Wilson R. Ciliary disorientation in patients with chronic upper respiratory tract inflammation. Am J Respir Crit Care Med 1995; 151: 800-804.

16. Sanchis J, Dolovich M, Rossman C, Wilson W, Newhouse M. Pulmonary mucociliary clearance in cystic fibrosis. $N$ Engl J Med 1973; 288: 651-654.

17. Rutland J, Cole PJ. Nasal mucociliary clearance and ciliary beat frequency in cystic fibrosis compared with sinusitis and bronchiectasis. Thorax 1981; 36: 654-658.

18. Kollberg H, Mossberg B, Afzelius BA, Philipson K, Camner P. Cystic fibrosis compared with the immotile-cilia syndrome. A study of mucociliary clearance, ciliary ultrastructure, clinical picture and ventilatory function. Scand J Respir Dis 1978; 59: 297-306.

19. Middleton PG, Geddes DM, Alton EWFW. Effect of amiloride and saline on nasal mucociliary clearance and potential difference in cystic fibrosis and normal subjects. Thorax 1993; 48: 812-816.

20. Armengot M, Escribano A, Carda C, Sanchez C, Romero C, Basterra J. Nasal mucociliary transport and ciliary ultrastructure in cystic fibrosis. A comparative study with healthy volunteers. Int J Ped Otorhinolaryngol 1997; 40: 2734.

21. Wood RE, Wanner A, Hirsch J, Farrell PM. Tracheal mucociliary transport in patients with cystic fibrosis and its stimulation by terbutaline. Am Rev Resp Dis 1975; 111: 733738.

22. Regnis JA, Robinson M, Bailey DL, et al. Mucociliary clearance in patients with cystic fibrosis and in normal subjects. Am J Respir Crit Care Med 1994; 150: 66-71.

23. Robinson $\mathrm{M}$, Eberl $\mathrm{S}$, Tomlinson $\mathrm{C}$, et al. Regional mucociliary clearance in patients with cystic fibrosis. $J$ Aero Med 2000; 13: 73-86.

24. Bateman JRM, Pavia D, Sheahan NF, Agnew JE, Clarke SW. Impaired tracheobronchial clearance in patients with mild stable asthma. Thorax 1983; 38: 463-467. 
25. Ogino $\mathrm{S}$, Nose $\mathbf{M}$, Irifune $\mathbf{M}$, Kikumori $\mathrm{H}$, Igarashi $\mathrm{T}$. Nasal mucociliary clearance in patients with upper and lower respiratory diseases. J Otorhinolaryngol Relat Spec 1993; 55: 352-355.

26. Mak GK, Henig NR. Sinus disease in cystic fibrosis. Clin Rev Allergy Immunol 2001; 21: 51-63.

27. Robinson M, Daviskas E, Eberl S, et al. The effect of inhaled mannitol on bronchial mucus clearance in cystic fibrosis patients: a pilot study. Eur Respir J 1999; 14: 678-685.

28. Kellerman D, Evans R, Mathews D, Shaffer C. Inhaled P2Y2 receptor agonists as a treatment for patients with cystic fibrosis lung disease. Adv Drug Deliv Rev 2002; 54: 14631474.

29. Konstan MW, Davis PB. Pharmacological approaches for the discovery and development of new anti-inflammatory agents for the treatment of cystic fibrosis. Adv Drug Deliv Rev 2002; 54: 1409-1423.

30. Rosenstein BJ, Cutting GR. The diagnosis of cystic fibrosis: a consensus statement. Cystic Fibrosis Foundation Consensus Panel. J Pediatr 1998; 132: 589-595.

31. Holzmann D, Ott PM, Felix H. Diagnostic approach to primary ciliary dyskinesia: a review. Eur J Pediatr 2000; 159: 95-98.

32. Engesaeth VG, Warner JO, Bush A. New associations of primary ciliary dyskinesia syndrome. Pediatr Pulmonol 1993; 16: 9-12.

33. Birch DS, Saleh HA, Wodehouse T, Simpson IN, Mackay IS. Assessing the quality of life for patients with chronic rhinosinusitis using the "Rhinosinusitis Disability Index". Rhinology 2001; 39: 191-196.

34. Stanley P, MacWilliam L, Greenstone M, Mackay I, Cole P. Efficacy of a saccharin test for screening to detect abnormal mucociliary clearance. Br J Dis Chest 1984; 78: 62-65.

35. Corbo GM, Foresi A, Bonfitto P, Mugnano A, Agabiti N, Cole PJ. Measurement of nasal mucociliary clearance. Arch Dis Child 1989; 64: 546-550.

36. Noah TL, Henderson FW, Wortman IA, et al. Nasal cytokine production in viral acute upper respiratory infection of childhood. J Infect Dis 1995; 171: 584-592.

37. Grubb BR, Jones JH, Boucher RC. Mucociliary transport determined by in vivo microdialysis in the airways of normal and CF mice. Am J Physiol Lung Cell Mol Physiol 2004; 286 : L588-L595.

38. Zahm JM, Gaillard D, Dupuit F, et al. Early alterations in airway mucociliary clearance and inflammation of the lamina propria in CF mice. Am J Physiol 1997; 272: C853C859.

39. McCray PB Jr, Zabner J, Jia HP, Welsh MJ, Thorne PS. Efficient killing of inhaled bacteria in DeltaF508 mice: role of airway surface liquid composition. Am J Physiol 1999; 277: L183-L190.

40. Robinson M, Bye PTB. Mucociliary clearance in cystic fibrosis. Ped Pul 2002; 33: 293-306.

41. Button B, Boucher RC. Cyclic compressive stress modulates ion transport in cystic fibrosis airway epithelial cell. Pediatr Pulmonol 2003; S25: 213.

42. Nixon GM, Armstrong DS, Carzino R, et al. Early airway infection, inflammation, and lung function in cystic fibrosis. Arch Dis Child 2002; 87: 306-311.

43. Konstan MW, Hilliard KA, Norvell TM, Berger M. Bronchoalveolar lavage findings in cystic fibrosis patients with stable, clinically mild lung disease suggest ongoing infection and inflammation. Am J Respir Crit Care Med 1994; 150: 448-454.

44. Talbot AR, Herr TM, Parsons DS. Mucociliary clearance and buffered hypertonic saline solution. Laryngoscope 1997; 107: 500-503

45. Noah TL, Black HR, Cheng PW, Wood RE, Leigh MW. Nasal and bronchoalveolar lavage fluid cytokines in early cystic fibrosis. J Infect Dis 1997; 175: 638-647.

46. Balfour-Lynn IM, Valman B, Silverman M, Webster AD. Nasal IgA response in wheezy infants. Arch Dis Child 1993; 68: 472-476.

47. Haslam PL, Baughman RP. Report of ERS Task Force: guidelines for measurement of acellular components and standardization of BAL. Eur Respir J 1999; 14: 245248.

48. Meeks M, Bush A. Primary ciliary dyskinesia (PCD). Pediatr Pulmonol 2000; 29: 307-316.

49. Narang I, Ersu R, Wilson NM, Bush A. Nitric oxide in chronic airway inflammation in children: diagnostic use and pathophysiological significance. Thorax 2002; 57: 586-589. 